\title{
Low-bond axisymmetric drop shape analysis for surface tension and contact angle measurements of sessile drops
}

\author{
Aurélien F. Stalder ${ }^{\mathrm{a}, *}$, Tobias Melchior $^{\mathrm{b}}$, Michael Müller $^{\mathrm{b}}$, Daniel Sage ${ }^{\mathrm{d}}$, Thierry Blu ${ }^{\mathrm{c}}$, Michael Unser ${ }^{\mathrm{d}}$ \\ a Department of Radiology, Xuanwu Hospital - Capital Medical University, Beijing, China \\ b Institute of Energy Research, Forschungszentrum Jülich GmbH, Jülich, Germany \\ ${ }^{c}$ Department of Electronic Engineering, Chinese University of Hong Kong, Hong Kong \\ d Biomedical Imaging Group, Ecole Polytechnique Fédérale de Lausanne (EPFL), Lausanne, Switzerland
}

\section{A R T I C L E I N F O}

\section{Article history:}

Received 16 March 2010

Received in revised form 28 April 2010

Accepted 29 April 2010

Available online 7 May 2010

\section{Keywords:}

Contact angle measurement

Surface tension measurement

Drop shape analysis

Sessile drop

ADSA

Coal ash slag

\begin{abstract}
A B S T R A C T
A new method based on the Young-Laplace equation for measuring contact angles and surface tensions is presented. In this approach, a first-order perturbation technique helps to analytically solve the Young-Laplace equation according to photographic images of axisymmetric sessile drops. When appropriate, the calculated drop contour is extended by mirror symmetry so that reflection of the drop into substrate allows the detection of position of the contact points. To keep a wide range of applicability, a discretisation of the drop's profile is not realised; instead, an optimisation of an advanced image-energy term fits an approximation of the Young-Laplace equation to drop boundaries. In addition, cubic B-spline interpolation is applied to the image of the drop to reach subpixel resolution. To demonstrate the method's accuracy, simulated drops as well as images of liquid coal ash slags were analysed. Thanks to the high-quality image interpolation model and the image-energy term, the experiments demonstrated robust measurements over a wide variety of image types and qualities. The method was implemented in Java and is freely available [A.F. Stalder, LBADSA, Biomedical Imaging Group, EPFL, http://bigwww.epfl.ch/demo/dropanalysis].
\end{abstract}

(c) 2010 Elsevier B.V. All rights reserved.

\section{Introduction}

\subsection{Problem description}

Surface tension and contact angles are consequences of intermolecular forces. The challenge is to understand how these short-range forces influence macroscopic behaviour of liquids. The fundamental equation of capillarity (1) relates the pressure gradient across a surface to its curvature. It has been given in 1805 by Young [2] and by Laplace [3].

$\Delta P=\gamma\left(\frac{1}{R_{1}}+\frac{1}{R_{2}}\right)$

In Eq. (1), $R_{1}$ and $R_{2}$ are the principal radii of curvature at any point on the drop, $\gamma$ is the surface tension and $\Delta P$ is the pressure difference across the surface.

As contact angles are a very local phenomenon, their direct measurement is limited by the resolution of the image acquisition system. Nevertheless, under certain conditions, it is possible

\footnotetext{
* Corresponding author. Fax: +41216933701.

E-mail address: aurelien.stalder@a3.epfl.ch (A.F. Stalder).
}

to apply a global drop model that can help to enhance accuracy and that allows for a derivation of surface tension.

\subsection{Available techniques for contact angle and surface tension measurements}

Several methods for contact angle and surface tension measurements have already been presented. Contact angles may be measured directly using a goniometer or may be calculated based on a polynomial fitting, an active contour (or snake) approach or a spherical approximation. Surface tension can be measured using the Wilhelmy plate method for example. But pendant or sessile drop methods [4] are now often combined with an axisymmetric drop shape analysis (ADSA) technique to provide both contact angles and surface tension based on the Young-Laplace equation.

\subsubsection{Goniometer measurement}

Due to its simplicity, the sessile drop method is the most widely employed procedure to measure contact angles. Direct determination using a goniometer on a telescope, a protractor on pictures or its computer-based equivalent can often be found [5]. The major drawback of these methods is subjectivity to operator's action. 


\subsubsection{Polynomial fitting}

In the polynomial fitting approach, a number of points from the contour of a drop near to the contact points are extracted and fitted by a polynomial. The degree of the polynomial as well as the number of coordinate points should be adjusted. The resulting contact angle, which is calculated using the polynomial's derivative, can be highly dependent on these parameters [6].

\subsubsection{Snake approach}

The more recent snake approach (DropSnake method [7]) unifies the global form of a drop and the locality of its contact angle. A snake is linked by elasticity constraints whose forces are of limited range depending on the order of the spline employed. Consequently, the snake is capable of revealing local phenomena such as contact angles while keeping trace of the global form of a drop. In addition, the global model allows for finding a symmetry in the image which can enhance detection of a drop's baseline and tilt angle.

\subsubsection{Spherical approximation}

The most obvious simplification of the Young-Laplace equation is approximating the solution by a sphere. Considering that sessile drops are mostly of small volume, action of forces such as gravity may often be neglected in comparison to surface tension effects. The corresponding approach treats $\Delta P$ as a constant in (1) and results in a surface with constant curvature, i.e. a sphere. By treating the contour as a sphere, one easily obtains a contact angle from a few points of the drop boundaries. Using a procedure known as $\theta / 2$-method, one calculates contact angles by only knowing base diameter and height of the sessile drop. As long as the spherical hypothesis is valid, this method yields plausible results [8].

\subsubsection{Axisymmetric drop shape analysis}

In many situations, neglecting gravity and using the spherical assumption is too far-fetched. The sessile drop method is employed if merely gravity is the dominating force. Under such conditions and given a horizontal and homogeneous substrate surface, the drop may be considered to be axisymmetric. If there are no forces applied other than gravity, the pressure difference varies linearly with the elevation according to Eq. (2) [4].

$\Delta P=\Delta P_{0}+\Delta \rho g h$

In Eq. (2), $\Delta P_{0}$ is the pressure gradient at a reference plane, $h$ stands for the elevation with respect to this plane, $\Delta \rho$ represents the difference in density of phases involved and $g$ is the gravitational constant.

For axisymmetric drops, the two principal radii of curvature are equal at the apex of the drop. Placing the reference plane at this point, one obtains Eq. (3).

$\Delta P_{0}=\frac{2 \gamma}{b}$

where, $b$ is the radius of curvature at the apex.

In such case, the profile of an axisymmetric sessile drop follows the well-known insoluble second-order differential equation of Young-Laplace (4).

$\gamma\left(\frac{1}{R_{1}}+\frac{1}{R_{2}}\right)=\Delta \rho g h+\frac{2 \gamma}{b}$

Or using the capillary constant $(c=\Delta \rho \cdot g / \gamma)$ :

$\left(\frac{1}{R_{1}}+\frac{1}{R_{2}}\right)=c h+\frac{2}{b}$

\subsubsection{Numerical integration of Young-Laplace equation}

The Young-Laplace equation for axisymmetric drops was first solved numerically in 1883 by Bashforth and Adams [9]. The authors tabulated solutions to (4) calculated by hand for different dimensionless Bond numbers $\beta=\Delta \rho \cdot g \cdot b^{2} / \gamma=c b^{2}$, which represent the deviation of the drop profile from a sphere. One may use these tables in order to identify the profile of a drop and get surface tension values as well as precise contact angles. This approach is fastidious and limited to a certain range of $\beta$. Thanks to computer algorithms, axisymmetric drop shape analysis effectuates the numerical integration automatically for any number of $\beta$ [10]. After discretising the drop contour in an image, it searches for the best corresponding Young-Laplace solution. As a result, an accurate contact angle and a value for $\beta$ are provided. Knowing the density of the liquid under investigation, one can deduce its surface tension from $\beta$. Although the ADSA method has been investigated thoroughly and is well-recognised today, deficiencies have been found when trying to identify surface tensions of nearly spherical drops [11]. This is due to the fact that for such shapes, significantly different surface tension values result in just slightly different drop profiles. As the ADSA procedure necessitates numerical integration of the Young-Laplace equation, it is computationally costly.

The ADSA contours were originally discretised using simple edge detectors like Sobel [12] or more advanced techniques such as Jensen-Shannon divergence based methods [13]. Depending on image characteristics and segmentation procedure, drop edges are detected more or less accurately. In some cases, especially when the image is blurred, such approaches are often subject to failure [14].

A variant of ADSA, called theoretical image fitting analysis (TIFA), deals with a continuously defined drop contour. It uses a gradient-based error function and is consequently able to better handle blurry images (e.g. captive bubbles) than the standard ADSA technique [14]. First, a theoretical gradient image is built using a numerical solution to the Young-Laplace equation. Then, the error function is defined as the sum of the squares of differences between an experimental gradient image and the theoretical one. In this approach, the contour is no longer discretised and the optimisation takes into account continuously defined gradient values. In another variant [15], a component labeling method was used to improve drop contour detection of noisy images. These techniques can extend the analysis of drop shapes to domains where approaches based on edge detectors fail because of drop images being too blurry or noisy.

\subsubsection{Analytical approximation solution}

In order to obtain a simpler solution to (4), perturbation theory may be employed. First use of perturbation theory in this context has been made by Ehrlich [16]. Using surface energy minimisation, Shanahan obtained a first-order solution to the profile of an axisymmetric drop [17]. Such solution allowed for the estimation of contact angles from the drop's height, maximum radius and contact radius.

\subsection{A new approach: low-Bond axisymmetric drop shape analysis (LBADSA)}

In the presented work, thanks to a judicious parametrisation, a small-perturbation solution to the contour of a sessile drop based on the Young-Laplace equation has been derived using basic analysis. Similar to the ADSA method, a theoretical profile obtained from the perturbation solution gets fitted to the drop contour.

Nevertheless, in LBADSA, the theoretical profile is not fitted to a discretised drop contour but is optimised based on an image energy approach. In this approach, segmentation and fitting are combined in what can be seen as a model-based segmentation. The complete pixel information is used during the fitting process. This approach is particularly advantageous when a clear accurate contour detection is difficult because of unsharp or noisy boundaries. Application of 
image energies to segmentation tasks is an active research domain. Following current proposals [18], a gradient image energy comprising gradient direction is used. This energy term has the benefit of being invariant to parametrisation [19]. In addition, it is suggested to account for pixel value information by a region energy [19]. Region energies are statistically based and offer the advantage of having a wide attraction range. The image energy approach has already been applied to the domain of contact angle measurements (DropSnake method [7]) and demonstrated its potential for noisy and/or low contrast data.

Finally, the presented method is applied to a continuous image of the drop by using cubic B-spline interpolation. Then, the evolution procedure takes place in this continuous domain to avoid inaccuracies introduced by pixelisation and discretisation.

\subsection{Application to surface tension measurements of coal ash slags}

LBADSA was used to analyse drop images of molten coal ash slags. Those slags play a major role in the design of $\mathrm{CO}_{2}$-emissionreduced power plants in which coal is gasified with the help of an appropriate reactor (Integrated Gasification Combined Cycle $[20,21])$. Due to extremely high temperatures in this gasification step, ash particles from the coal occur in liquid form (slag) and represent a danger for the subsequent gas turbine process.

Removal of ash particles from the synthesis gas leaving the gasification reactor should be performed at highest possible temperatures in order to guarantee good efficiencies of the integrated cycle [22]. This can be achieved by introducing ceramic spheres into the gas' flow path on which slags deposit [23]. For the correct choice of ceramics as well as for an appropriate reactor design, physicochemical properties of coal ash slags need to be known. One of such characterising properties is surface tension which was studied using a sessile drop inside a high-temperature furnace.

The particular imaging system of the experimental facility sometimes produces images with limited contrast that are difficult to analyse using standard drop detection algorithms. In addition, drop contours may appear blurred due to heat radiation. To compensate for such deficiencies and to validate the measurement results with regard to analysis algorithms, all drop images were processed by LBADSA in addition to two other software packages.

\section{Methodology}

\subsection{Drop contour detection}

\subsubsection{Unified image energy}

A drop contour detection scheme based on unified gradient and region energies [19] is used. The combination allows for both preciseness and large convergence radius and was already applied in the snake approach to drop contour detection [7].

The gradient-based image energy is given by:

$E_{\text {edge }}=\oint_{C} k \cdot(\nabla f(r) \times d r)$

where $k$ denotes the unit orthogonal vector to the image plane and $\nabla f(r)$ is the gradient of the image $f$ at point $r$ of curve $C$.

Using Green's theorem (6) is expressed as surface integral (7) where $\nabla$. is the divergence operator.

$E_{\text {edge }}=\iint_{S} \underbrace{\nabla \cdot \nabla f(s)}_{T_{e}(f)} d s$
The region energy is given by:

$E_{\text {region }}=\iint_{S} T_{r}(f) d s$

where the pixel intensity distribution $T_{r}(f)$ is required. Considering that sessile drop images are often produced in dedicated environments under standard lighting conditions, this distribution can be determined only once. If pixel distribution is unknown, an estimation can be taken from an approximate contour during optimisation.

Having expressed gradient energy as a surface integral, the unified image energy is obtained according to Eq. (9) with $f_{u}=$ $m_{e} T_{e}(f)+\left(1-m_{e}\right) T_{r}(f)$.

$E_{\text {image }}=\iint_{S} f_{u}(s) d s$

where $0 \leq m_{e} \leq 1$ is the weight of the gradient term in the image energy and can be adjusted based on the application and image properties.

Applying Green's theorem again, this unified energy is rewritten as contour integral

$E_{\text {image }}=\oint_{C} f_{u}^{y}(x, y) d x=-\oint_{C} f_{u}^{x}(x, y) d y$

where

$f_{u}^{y}(x, y)=\int_{-\infty}^{x} f_{u}(x, \tau) d \tau$

$f_{u}^{x}(x, y)=\int_{-\infty}^{y} f_{u}(\tau, y) d \tau$

\subsubsection{Image interpolation}

The discrete nature of drop images may influence contact angle and surface tension measurements [24]. In order to minimise the influence of discretisation, cubic B-spline interpolation is used based on its excellent trade-off between interpolation quality and computational burden [25].

\subsection{First-order perturbation solution to Young-Laplace equation}

Starting from the Young-Laplace equation for axisymmetric drops (5), an approximate solution is now developed using firstorder perturbation theory. The motivation is to obtain a closed form solution which is not available in the general case.

\subsubsection{Coordinate system}

Fig. 1 represents the meridian profile of an axisymmetric drop resting on a horizontal surface. Defining the angle $\alpha$ with respect to the drop's apex, the origin of this parametrisation is situated at a distance $b$, being named radius of curvature at the apex. The profile can thus be expressed according to:

$\left\{\begin{array}{l}x=r(\alpha) \sin \alpha \\ z=-r(\alpha) \cos \alpha\end{array}\right.$

Under the assumption of axial-symmetry $r(-\alpha)=r(\alpha)$, curvatures indices in this new parametrisation are calculated as follows:

$\frac{1}{R_{1}}=\frac{r^{2}+2 r^{\prime 2}-r r^{\prime \prime}}{\left(r^{\prime 2}+r^{2}\right)^{3 / 2}}$

$\frac{1}{R_{2}}=\frac{1-\left(r^{\prime} / r \tan \alpha\right)}{\left(r^{\prime 2}+r^{2}\right)^{1 / 2}} \quad \forall \quad z^{\prime} \geq 0$

The assumption for deriving (15) is that the center of curvature of the drop's surface in the plane normal to both surface and meridian 


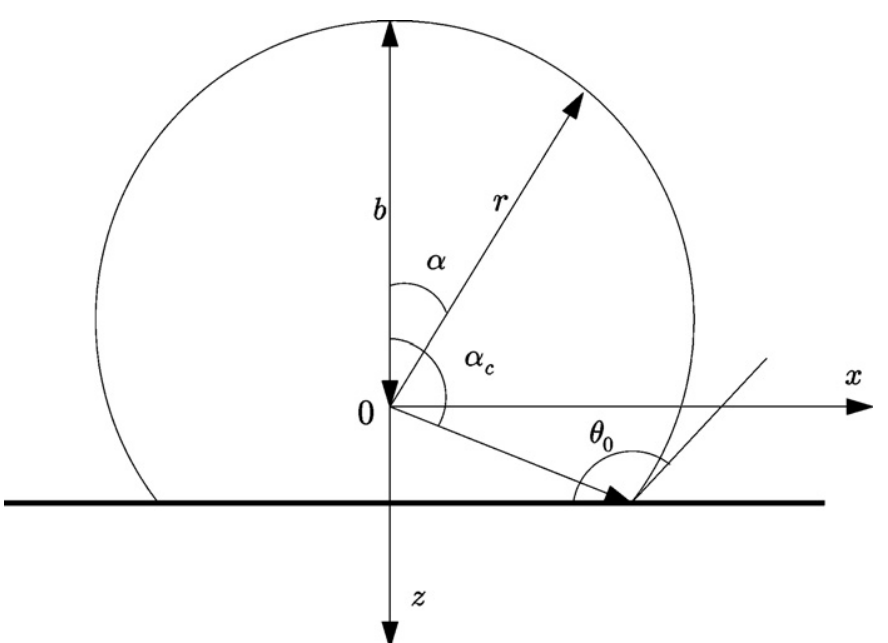

Fig. 1. Definition of a coordinate system for an axisymmetric drop resting on a horizontal surface.

section is situated on the drop's revolution axis. In this parametrisation, the Young-Laplace equation for axisymmetric drops (5) transforms into:

$\frac{1}{R_{1}}+\frac{1}{R_{2}}=\frac{2 r^{2}+3 r^{\prime 2}-r r^{\prime \prime}-(1 / \tan \alpha)\left(\left(r^{\prime}\right)^{3} / r+r^{\prime} r\right)}{\left(r^{\prime 2}+r^{2}\right)^{3 / 2}}=c(b-r \cos \alpha)+\frac{2}{b}$

where $h=b+z=b-r \cos \alpha$.

\subsubsection{Small-perturbation theory}

Assuming that the drop profile deviates only slightly from a circle, a small-perturbation approach

$r=b(1+\varepsilon p(\alpha))$

where it is assumed that $\varepsilon \ll 1$ and $p(\alpha)$ has sufficient regularity so that $|p(\alpha)|<1,\left|p^{\prime}(\alpha)\right|<1$ and $\left|p^{\prime \prime}(\alpha)\right|<1$.

Introducing the first and second derivative of (17) with respect to $\alpha$ into (16) and factoring out $b$, we obtain:

$$
\begin{gathered}
2(1+\varepsilon p)^{2}+3\left(\varepsilon p^{\prime}\right)^{2}-(1+\varepsilon p) \varepsilon p^{\prime \prime}-\frac{1}{\tan \alpha}\left(\frac{\left(\varepsilon p^{\prime}\right)^{3}}{1+\varepsilon p}+(1+\varepsilon p) \varepsilon p^{\prime}\right) \\
-\left(c b^{2}(1-\cos \alpha(1+\varepsilon p)+2)\left(\left(\varepsilon p^{\prime}\right)^{2}+(1+\varepsilon p)^{2}\right)^{3 / 2}=0\right.
\end{gathered}
$$

The terms in $\varepsilon^{2}$ are then neglected, which results in the new simplified second-order differential equation:

$-\varepsilon p^{\prime \prime}(\alpha)-\varepsilon \frac{p^{\prime}(\alpha)}{\tan \alpha}+\left(-2+c b^{2}(-3+4 \cos \alpha)\right) \varepsilon p(\alpha)+c b^{2}(\cos \alpha-1)=0$

\subsubsection{Assumption on $c b^{2}$}

Contact angle and surface tension measurements are generally accomplished using small drops whose diameters are of the order of magnitude of millimetres. Observing that capillary constants $c$ are of the order of magnitude of $10^{5} \mathrm{~m}^{-2}$ (Table 1 ), it may be inferred that $c b^{2} \ll 1$, thus allowing for the simplification $-2+c b^{2}(-3+4 \cos \alpha) \cong-2$.

Table 1

Calculated capillary constants based on surface tension and density data [26] for selected liquids.

\begin{tabular}{llcl}
\hline & $\gamma\left[\mathrm{N} \mathrm{m}^{-1}\right]$ & $\rho\left[\mathrm{kg} \mathrm{m}^{-3}\right]$ & $c\left[\mathrm{~m}^{-2}\right]$ \\
\hline Dodecane & 0.025 & 750 & 294,300 \\
Diiodomethane & 0.0514 & 3325 & 634,667 \\
Mercury & 0.47 & 13,534 & 282,486 \\
Water & 0.0728 & 1000 & 134,753 \\
\hline
\end{tabular}

Defining $C=c b^{2} / \varepsilon$, the following nonhomogeneous differential equation of second order with non-constant coefficients is deduced:

$p^{\prime \prime}(\alpha) \sin \alpha+p^{\prime}(\alpha) \cos \alpha+2 p(\alpha) \sin \alpha-C(\cos \alpha-1) \sin \alpha=0$

\subsubsection{Solution}

Using $p(0)=0$ as boundary condition, Eq. (20) may be solved analytically (see supplementary data online) leading to Eq. (21) which is a first-order small-perturbation solution to (16).

$r(\alpha)=b(1+\varepsilon p(\alpha))=b+\frac{c b^{3}}{3}\left[\cos \alpha\left(-\ln |1+\cos \alpha|+\ln 2+\frac{1}{2}\right)-\frac{1}{2}\right]$

The contact angle $\alpha_{c}$ (see Fig. 1) can be deduced by geometrical considerations according to (22).

$\tan \left(\pi-\theta_{0}\right)=\left.\frac{z^{\prime}}{x^{\prime}}\right|_{\alpha_{c}}=\frac{r\left(\alpha_{c}\right) \sin \alpha_{c}-r^{\prime}\left(\alpha_{c}\right) \cos \alpha_{c}}{r\left(\alpha_{c}\right) \cos \alpha_{c}+r^{\prime}\left(\alpha_{c}\right) \sin \alpha_{c}}$

\subsubsection{Parametrisation}

Using the previously derived first-order perturbation solution, the drop profile may be described by expression (23). Here, $r(\alpha)$ is given by (21) whereas $x_{0}$ and $z_{0}$ are the coordinates of the drop's apex.

$\left\{\begin{array}{l}x=x_{0}+r(\alpha) \sin \alpha \\ z=z_{0}+b-r(\alpha) \cos \alpha\end{array}\right.$

The reflected profile is defined by a horizontal symmetry at $z=$ $z_{h}=z\left(\alpha_{c}\right)$, it is provided by Eq. (24).

$\left\{\begin{array}{l}x_{r}=x_{0}+r(\alpha) \sin \alpha \\ z_{r}=2 z_{h}-\left(z_{0}+b-r(\alpha) \cos \alpha\right)\end{array}\right.$

\subsection{Contour optimisation}

\subsubsection{Energy derivatives}

Using the drop parametrisation defined in Fig. 1, image energy (see Section 2.1) is given by expressions (25) and (26).

$$
\begin{aligned}
& E_{\text {image }}=\int_{0}^{\alpha_{c}}\left[f_{u}^{z}(x(\alpha), z(\alpha)) x^{\prime}(\alpha)+f_{u}^{z_{r}}\left(x_{r}(\alpha), z_{r}(\alpha)\right) x_{r}^{\prime}(\alpha)\right] d \alpha \\
& E_{\text {image }}=-\int_{0}^{\alpha_{c}}\left[f_{u}^{\chi}(x(\alpha), z(\alpha)) z^{\prime}(\alpha)+f_{u}^{x_{r}}\left(x_{r}(\alpha), z_{r}(\alpha)\right) z_{r}^{\prime}(\alpha)\right] d \alpha
\end{aligned}
$$

The image energy in (25) and (26) is combined with the perturbation solution in order to deduce partial derivatives of the image energy (see supplementary data online). The partial derivatives are required for the optimisation algorithm.

\subsubsection{Computation of energy derivatives}

The image energy's partial derivatives (see supplementary data online) appear to induce a considerable amount of computational burden as they involve integrals and trigonometric functions. But the same trigonometric functions occur in $r(\alpha), \partial r / \partial b, \partial r / \partial c$ as well as in $r^{\prime}(\alpha), \partial r^{\prime} / \partial b, \partial r^{\prime} / \partial c$ whereas $\alpha$ is bounded to $-\pi \leq \alpha \leq \pi$ or even to $0 \leq \alpha \leq \pi$ if symmetry is respected. Pre-calculation of trigonometric functions does therefore not necessitate much memory and all integrals are approximated by finite sums during the optimisation process.

\subsubsection{Optimisation strategy}

A modular multivariable optimisation scheme based on the steepest descent algorithm is implemented in order to minimise the error function ((25) and (26)). It takes profit of analytical derivatives after normalisation based on a standard maximum step. 


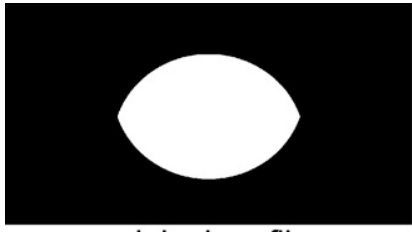

original profile

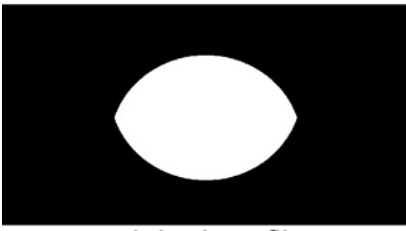

original profile

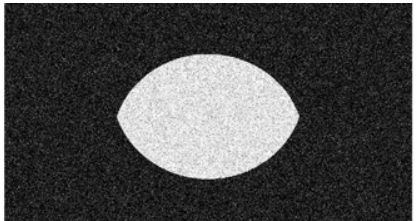

noise rel. variance $=0.1$

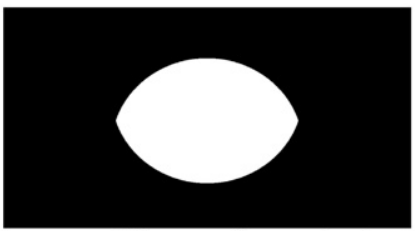

smoothing radius $=1 \mathrm{pix}$

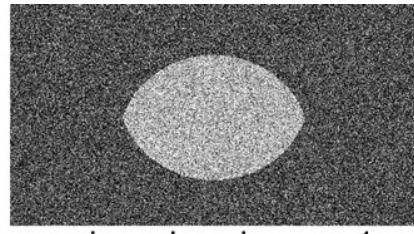

noise rel. variance $=1$

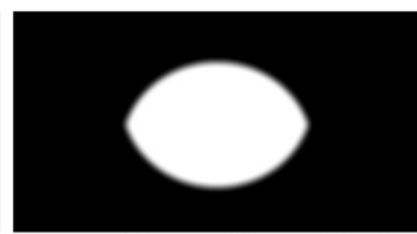

smoothing radius $=10$ pix

Fig. 2. Samples of a drop contour before and after application of noise and smoothing filters.

\subsection{Synthetic data}

Synthetic datasets were created by performing numerical integration of the Young-Laplace equation. Six drop contours based on different drop volumes $\left(20-60 \mathrm{~mm}^{3}\right)$, capillary constants $\left(0-196,200 \mathrm{~m}^{-2}\right)$ and contact angles $\left(45.5-161.1^{\circ}\right)$ were calculated. Drop profiles were subsequently altered by means of a gaussian smoothing filter and a gaussian noise filter. Smoothing was linearly incremented in 100 steps up to a smoothing radius of 10 pixels. Analogously, noise was incremented in 100 steps up to a gaussian noise variance equal to the dynamic range of the image. Sample images are displayed in Fig. 2 for one drop contour corresponding to a contact angle of $71.4^{\circ}$.

\subsection{Experimental setup}

The experimental facility employed for surface tension measurements of coal ash slags complies with a typical sessile drop setup [27-30]. An outline of the arrangement is shown in Fig. 3. It mainly consists of a high-temperature furnace, a CCD camera being attached to a zoom lens and an analysis computer which is equipped with a framegrabber to digitise drop images. The furnace permits to heat up a sample to $1500^{\circ} \mathrm{C}$ in a well-defined gas atmosphere at atmospheric pressure. By melting a cylindrical pellet ( $5 \mathrm{~mm}$ in diameter, about $5 \mathrm{~mm}$ in height) of ash to be studied, a drop is created on a certain substrate material. This drop is then photographed and resulting greyscale images are analysed by three different algorithms.

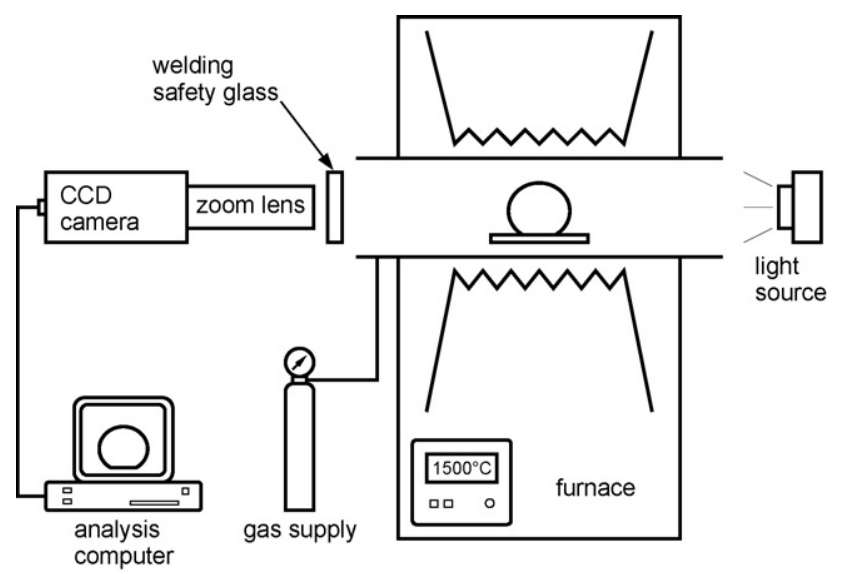

Fig. 3. Experimental facility used for surface tension measurements.
A welding safety glass is mounted in front of the zoom lens to guarantee a clear view of the furnace's interior. Until now, no image calibration step is incorporated into the measurement process, the zoom lens manufacturer states an optical distortion of up to $0.113 \%$ for its device. To allow for a calculation of image resolution, a reference object of known diameter is positioned next to the drop.

From the melting point of ash onwards, the furnace is heated at a rate of $2^{\circ} \mathrm{C} / \mathrm{min}$. Drop images are saved every $2^{\circ} \mathrm{C}$ to bitmap files. The brightness and contrast settings of the framegrabber are continuously adjusted by hand to compensate for changes in heat radiation due to an increase in temperature. Because none of the ashes studied so far forms a drop at temperatures below $1200^{\circ} \mathrm{C}$, radiation of the sample is fully sufficient to have a clear view onto the drop contour. Therefore, use of the light source is only made when aligning the sample inside the furnace at room temperature.

\subsection{Details on usage of LBADSA}

In order to analyse image series, a modified version of LBADSA was used. The analysis is initiated by manually providing parameters for the Young-Laplace approximation so that the photographed drop shape in the first image of a series is roughly represented by it. LBADSA then calculates a converged contour fit and passes results to the following image as initialisation solution. This process is repeated until the last image of a series is reached.

A contour fit is considered converged, when one iteration step results in contact angle and capillary constant variations of less than $10^{-6 \circ}$ and $10^{-6} \mathrm{~m}^{-2}$, respectively. If such convergence criteria are not achieved for an image, the analysis process is aborted after 300 iterations. Under these circumstances, both residuals were sometimes observed to fluctuate at values of about $10^{-5 \circ}$ and $10^{-5} \mathrm{~m}^{-2}$ without decreasing any longer.

A Gaussian smoothing filter of a 3.0 pixels radius is used for all measurements. The parameter $d$, standing for the size in pixels of drop reflection into substrate material, is constantly set to 1 as there is no reflection in current images. Based on experience, $d=1$ pixel leads to better substrate line detection by LBADSA than choosing $d=0$ in such cases.

\subsection{Other contact angle and surface tension determination algorithms}

In addition to LBADSA, two commercial algorithms that do not rely on image energies were used to analyse obtained drop pictures. Both codes implement a fitting of the numerically integrated exact Young-Laplace equation to the drop profile and can there- 
fore be considered as "classical" approaches in the light of works by Bashforth and Adams [9]. Among other values, all algorithms output contact angle, drop volume and surface tension being represented by either capillary constant $c=\Delta \rho \cdot g / \gamma$ or Bond parameter $\beta=\Delta \rho \cdot g \cdot b^{2} / \gamma$

Each drop picture is analysed independently using the commercial programs and a manual specification of the drop base line is followed by the contour fit.

The first algorithm - being called "SCA20" hereafter - is developed by DataPhysics in Germany [31] and offers support for communication with contact angle measuring devices sold by the company.

The second algorithm will be named "ADSA" in the further course of this article and originates from the University of Toronto. It looks for a black drop on a white background wherefore greyscale values of obtained images need to be inverted prior to analysis (see Figs. 5 and 7). Applications and discussions of this code can frequently be found in literature $[12,11,27,32,33]$.

Like with LBADSA, surface tension data needs to be calculated from capillary constant $c$ or Bond parameter $\beta$ when using the commercial codes.

\subsection{Processing of calculation results}

Due to some aberrant measurements, three filters needed to be introduced in order to check outputted datasets of all three algorithms for physical plausibility. The first such threshold restricts the contact angle to the range between $0^{\circ}$ and $180^{\circ}$. Secondly, the capillary constant is constrained to positive values which assures $c \neq 0$ for a correct calculation of surface tension. Thirdly, values for $\gamma$ are limited to the interval of $0 \mathrm{mN} / \mathrm{m}$ to $1500 \mathrm{mN} / \mathrm{m}$ complying with literature specifications for coal ash slags [34-36,28,37]. Datasets are discarded completely if one of the presented filter conditions fails. In order to obtain surface tension values from output data, capillary constant $c$ and drop volume $v$ have to be used. By dividing an average sample mass $\bar{m}$ determined before and after the experiment by the calculated drop volume, surface tension $\gamma$ is derived according to $\gamma=\bar{m} \cdot g / v \cdot c$. It has to be noted that the density of the surrounding gas atmosphere is neglected in this approach. In addition, errors in drop volume calculation and mass determination directly affect the resulting surface tensions.

This article solely presents data points that successfully passed the filter conditions for all three algorithms.

\section{Results}

\subsection{Simulated drop profiles}

Fig. 4 shows drop profiles obtained from numerical integration of the Young-Laplace equation, analytical approximation and spherical approximation. All contours correspond to a drop with a large apex radius $(3 \mathrm{~mm})$ and a contact angle of $180^{\circ}$. These parameters were chosen to show the error due to the approximation that appears for large drops and large contact angles. Capillary constants were augmented from $c=0 \mathrm{~m}^{-2}$ to $c=400,000 \mathrm{~m}^{-2}$ (water $\approx 130,000 \mathrm{~m}^{-2}$, mercury $\left.\approx 280,000 \mathrm{~m}^{-2}\right)$. For $c=0$, all curves overlap completely but as capillary constant increases, drop profiles of spherical approximation deviate considerably from the Young-Laplace shape. In spite of the large apex radius, drop contours of the analytical approximation differ slightly from the numerical solution. Discrepancies are solely present for large capillary constants, near to the interface and for large contact angles.

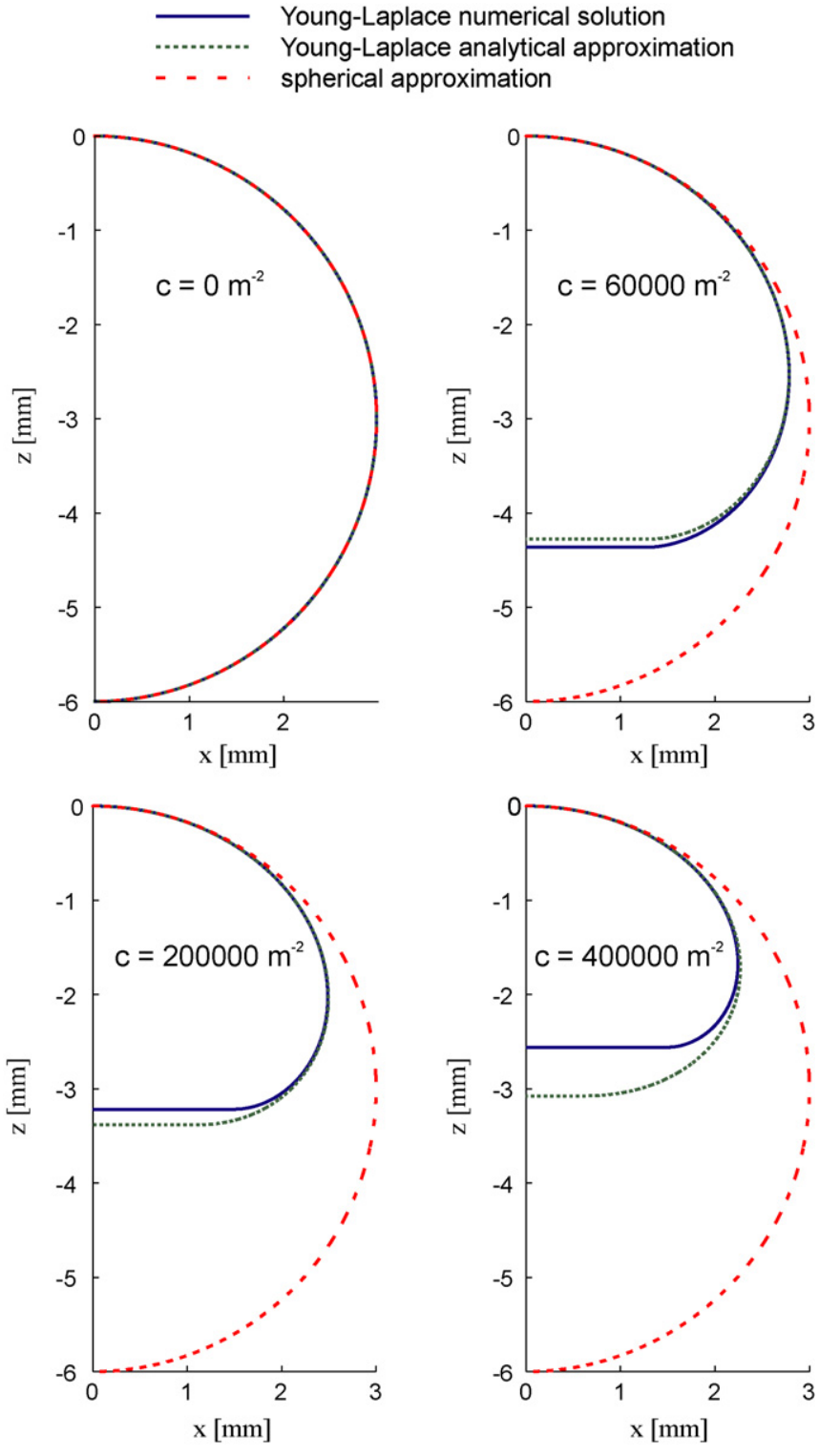

Fig. 4. Half drop profiles obtained from numerical integration of the Young-Laplace equation, analytical approximation solution and spherical approximation for a large drop with $3 \mathrm{~mm}$ apex radius, $180^{\circ}$ contact angle and various capillary constants.

\subsection{Synthetic drops}

The contact angle determination success rate on synthetic datasets after application of noise and smoothing filters is given in Table 2. All algorithms provide accurate contact angle detection on data without alteration due to smoothing or noise. While all codes can measure contact angles for low smoothing filters, SCA20 (93.8\%) and LBADSA (99.2\%) are most robust when blurry contours occur. In presence of noise, LBADSA is the only algorithm to perform contact angle measurements. Although the success rate is not as high as with blurry data, LBADSA is able to measure accurate

\section{Table 2}

Success rate of LBADSA, ADSA and SCA20 contact angle determination on synthetic datasets after application of smoothing and noise. Datapoints with less than $7 \%$ error were considered successful.

\begin{tabular}{llll}
\hline & LBADSA (\%) & ADSA (\%) & SCA20 (\%) \\
\hline Smoothing & 99.2 & 28.0 & 93.8 \\
Noise & 86.8 & 0 & 0 \\
\hline
\end{tabular}




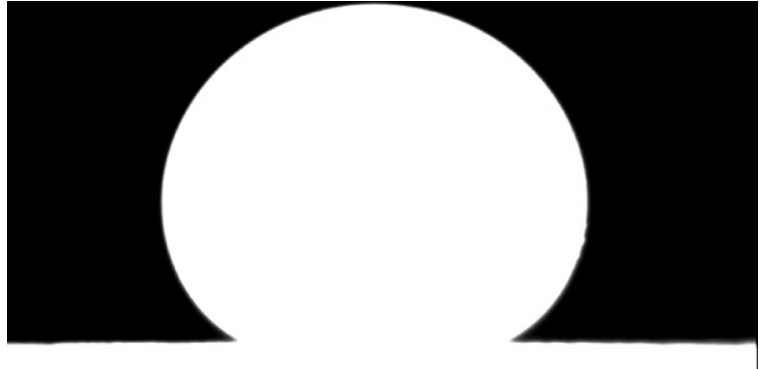

Fig. 5. German black coal ash on graphite at $1310^{\circ} \mathrm{C}$.

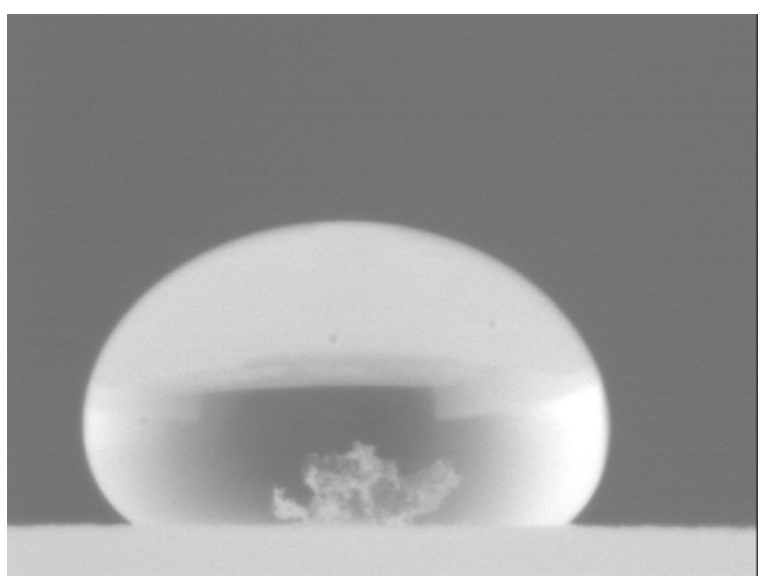

Fig. 6. $\mathrm{NaCl}$ on graphite at $860^{\circ} \mathrm{C}$.

contact angles in $86.8 \%$ of the trials. In all cases, accuracy of contact angle measurements decreased as the smoothing or noise filter's intensity increased.

\subsection{Experimental drops}

During sessile drop measurements, mainly two different drop types were observed. Depending on substrate material, surrounding gas atmosphere and ash under investigation, upright round drops (Figs. 5 and 6) or flat drops (Fig. 7) can be found. The applied algorithms generally perform better on large round drops, yielding low-scattering surface tension values as a function of temperature. This finding is in full accordance with literature [27].

The ratio of plausible datapoints produced by the three algorithms is shown in Table 3. Unlike Table 2, Table 3 does not indicate

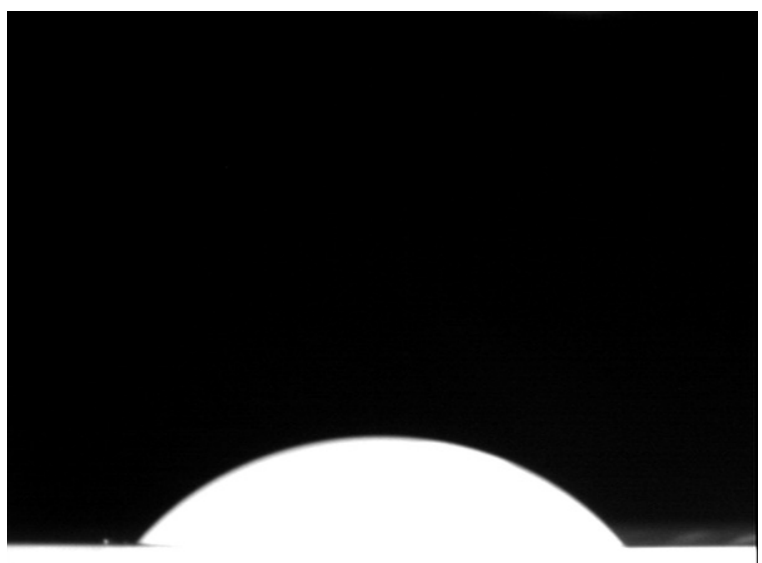

Fig. 7. German brown coal ash on platinum-gold alloy at $1420^{\circ} \mathrm{C}$.
Table 3

Ratio of plausible datapoints measured by LBADSA, ADSA and SCA20 in Figs. 5-7. A ratio of plausible datapoints below $100 \%$ indicate that in some situations either the algorithm failed to produce any result or that the result was aberant and excluded by the plausibility filter.

\begin{tabular}{llll}
\hline & LBADSA (\%) & ADSA (\%) & SCA20 (\%) \\
\hline Serie from Fig. 5 & 91.0 & 86.5 & 59.5 \\
Serie from Fig. 7 & 99.0 & 71.8 & 99.0 \\
Serie from Fig. 6 & 89.8 & 6.8 & 98.3 \\
\hline
\end{tabular}

the accuracy of the measurements but only the ability of each algorithm to measure data that are not aberrant. The plausible datapoints include all the measurements that could actually be performed by one algorithm (some images can make one algorithm fail and produce no measurements) and that were not excluded by the plausibility filter.

While ADSA and SCA20 presented a wide range of ratio of plausible datapoints on different datasets (from 6.8\% to 99.0\%), LBADSA coherently presented a high ratio of plausible datapoints $(\geq 89.8 \%)$. Nevertheless, Table 3 is not enough to assess the accuracy of the algorithms as one algorithm may gives out plausible yet inaccurate values.

To underline previous statements, Fig. 8 provides calculated contact angle and surface tension data for a coal ash forming a round drop. Fig. 9 illustrates results for the image series of a flat drop in contrast. Whereas surface tension scatters far less when round drops are investigated, contact angle calculation exhibits an opposite trend. Analysis of the flat drop image series leads to nearly constant contact angle gradients for all three computer codes. While the ADSA algorithm yields equally smooth contact angle curves for round drops, LBADSA and SCA20 show scattering results in those cases. This is likely due to the detection of the drop baseline. While ADSA and SCA20 are not able to detect the interface by themselves and use a constant level (manually defined) instead, LBADSA automatically detects the baseline in every image.

Common for nearly all round-drop-forming ashes are negative surface tension gradients at low temperatures and slightly rising or stagnating surface tensions at high temperatures (with respect to ADSA and LBADSA data). The increase of surface tension as well as the order of magnitude of surface tension values are in accordance with literature [34-36,28,37]. From Fig. 8 it is obvious that SCA20 outputs most deviating results whereas ADSA and LBADSA are in very good agreement with each other regarding surface tension. In addition, SCA20 yields less valid data points (after filtering) compared to other codes.

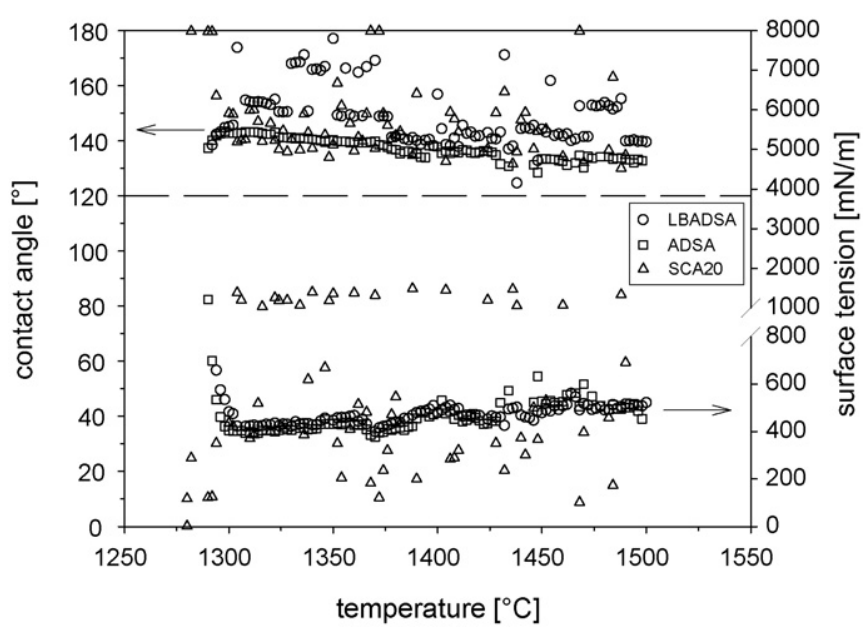

Fig. 8. Contact angle and surface tension for the round drop measurement series corresponding to Fig. 5. 


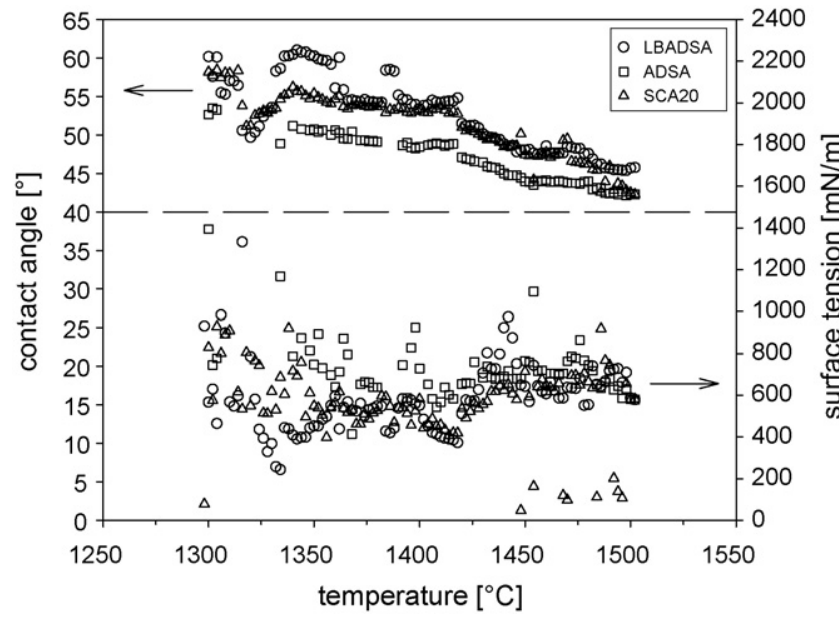

Fig. 9. Contact angle and surface tension for the flat drop measurement series corresponding to Fig. 7.

In Fig. 10, low-scattering contact angles and surface tensions can be found based on reference measurements using $\mathrm{NaCl}$ as melted material. Due to lower temperatures in these experiments, the black-white-gradient is much less pronounced than presented in Figs. 5 and 7. This effect also reduces the sharpness of acquired drop images (see Fig. 6). NaCl additionally forms wider drops compared to coal ash slags which may be the reason for improvement in SCA20's results $[38,39]$. In this lower contrast situation, ADSA often failed analysing the contour. On the contrary, LBADSA does not seem to be influenced by lower image quality at all. The continuous line shown in Fig. 10 denotes literature values for the surface tension of molten $\mathrm{NaCl}[40]$.

Fig. 11 gives reasons for negative surface tension gradients mentioned above (Fig. 8) by showing drop volume and capillary constant as a function of temperature for the upright round sessile drop in Fig. 5. The diagram states almost constant drop volumes at low temperatures, therefore, rising capillary constants are responsible for a decrease in surface tension. It should be kept in mind that drop volume and capillary constant are direct output values of computer codes whereas density and surface tension are indirectly calculated during post-processing. The sudden change in volume and capillary constant at about $1360^{\circ} \mathrm{C}$ can be explained by a slight collapse of the drop observable in acquired images (release

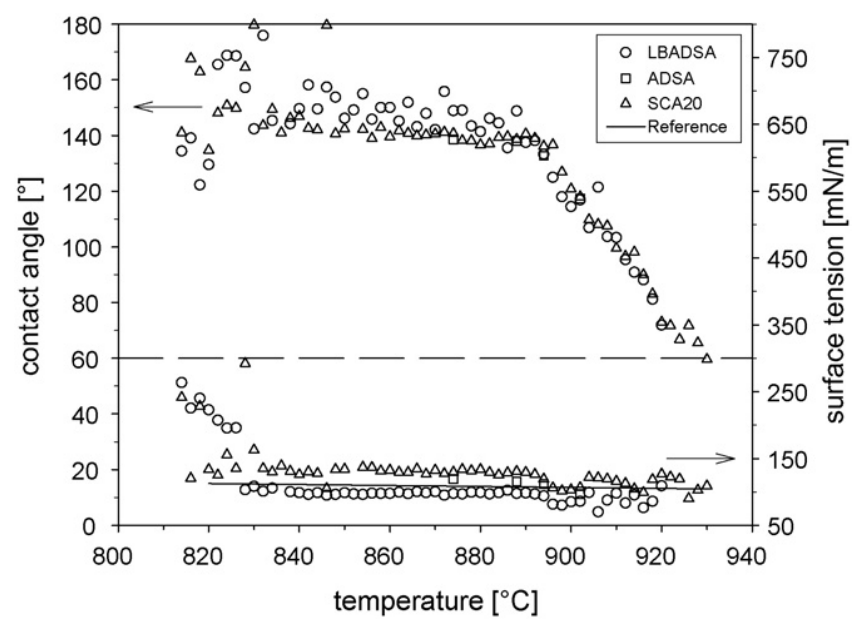

Fig. 10. Contact angle and surface tension for the round $\mathrm{NaCl}$ drop measurement series corresponding to Fig. 6.

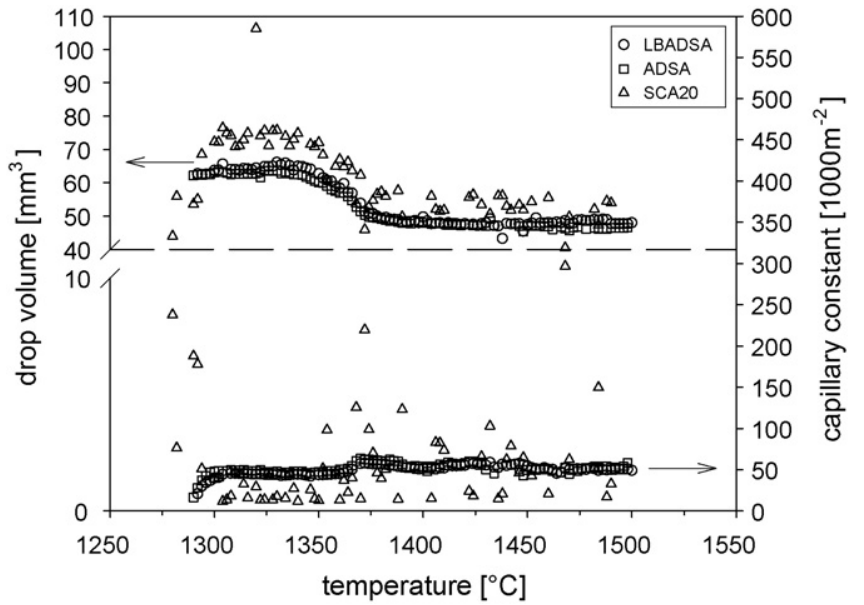

Fig. 11. Drop volume and capillary constant for the round drop measurement series corresponding to Figs. 5 and 8.

of gaseous species formed within the sessile drop due to chemical reactions of the slag).

\section{Discussion}

\subsection{Error due to first-order approximation in LBADSA}

Drop shape analysis using a first-order approximation of the Young-Laplace equation was accurate in most situations. The approach seems to be valid for many applications of the sessile drop method where drops have small volumes and capillary constants. Nevertheless in some situations, the approximation $c b^{2} \ll 1$ might limit the application of the method. When the approximation is no more valid, discrepancies can appear for large drops (i.e. large apex radius) with large capillary constants and large contact angles (see Fig. 4). Nonetheless, it is fairly easy to realise a rapid estimation of the approximation $c b^{2} \ll 1$ and hence verify prospectively or retrospectively the validity of calculations. In situations where the approximation is not valid any more, it could be possible to refine the output based on the analytical solution by using numerical integration of the Young-Laplace equation during the last optimisation steps.

\subsection{Comparison of algorithms}

Analysing a sample series composed of 100 drop images (comparable to Fig. 5) on a desktop PC took approximately 26 min for LBADSA, 2 min for ADSA and 1 minute for SCA20. At first sight, the analytical solution to the Young-Laplace equation realised in LBADSA should require less computation than a numerical approach. However, reduction of computational cost in drop contour calculation is overbalanced by using image energies and interpolation methods. The longer analysis time of LBADSA can furthermore be explained by the Java implementation as a plugin for the open-source software ImageJ [41]. As LBADSA is not optimised for speed yet, considerable calculation time improvements should be possible.

In order to yield sensible results, classical algorithms need to detect the photographed drop contour correctly, particularly near the substrate line [32]. This implies a high image contrast and clearly defined contours in a drop's contact point regions. Due to heat radiation (reflection) and setup of the measurement facility, such clearness could not always be assured during current investigations. In this context, LBADSA proves to be much more robust thanks to the use of image energies. It provides most reliable results 
for synthetic images altered with smoothing and noise as well as for low-contrast experimental photographs.

The current implementation of LBADSA is able to accurately detect a drop's baseline location in presence of reflection. This information helps to perform a precise drop analysis in turn. In absence of reflection, like for coal ash slags, automatic detection is not always correct and may introduce contact angle scattering (e.g. for round drops). Such situations might better be managed by manually defining the interface level. However, this feature is currently available for analysis of single images only and not for analysis of image series. Enhancing the interface detection procedure or allowing for manual adjustment can thus improve contact angle accuracy and reduce data scatter.

The current version of LBADSA uses a simple steepest descent algorithm. As for any optimisation method, solutions are dependent on initialisation. It can be assumed that more advanced optimisation strategies, such as Levenberg-Marquardt, can further enhance convergence radius, accuracy and speed.

\subsection{Application to coal ash slags}

The investigation of coal ash slag surface tensions as a function of temperature reveals a decrease in the melting interval of ashes. Subsequently, a temperature-dependent, slight rise in surface tension can be detected. Observed surface tension values are comprised between $400 \mathrm{mN} / \mathrm{m}$ and $800 \mathrm{mN} / \mathrm{m}$.

\section{Conclusions}

A new drop shape analysis method (LBADSA) allowing for calculation of contact angles and surface tensions from sessile drop images is presented. A first-order approximation of the Young-Laplace equation is used to provide an analytical solution to the contour of sessile drops at low computational burden. The economisation of machine time due to such analytical term allows for implementing a computationally intensive image energy approach. Unlike common drop shape analysis algorithms, the combined image energy technique does not discretise drop contours prior to fitting the Young-Laplace equation but employs full information on original pixel values throughout the optimisation process. Although LBADSA represents a first-order approximation solution to the Young-Laplace equation, the method is valid in most applications of the sessile drop technique where small drops are used. Furthermore, accounting for drop reflection in the code guarantees automatic interface detection and refined shape analysis as long as a drop mirror image is visible. The method is implemented as a plugin for the open-source software ImageJ and is freely available [1]. LBADSA was successfully validated on synthetic images altered with smoothing and noise as well as on experimental data from molten coal ash slags with a wide range of contrast properties. Compared to two commercially sold softwares, LBADSA provides a very broad range of applicability with respect to different image types and quality.

\section{Appendix A. Supplementary data}

Supplementary data associated with this article can be found, in the online version, at doi:10.1016/j.colsurfa.2010.04.040.

\section{References}

[1] A.F. Stalder, Biomedical Imaging Group of École Polytechnique Fédérale de Lausanne, Drop Shape Analysis-Free Software for High Precision Contact Angle Measurement, last checked: December 13th, 2009, URL http://bigwww.epfl.ch/ demo/dropanalysis.

[2] T. Young, An essay on the cohesion of fluids, Phil. Trans. R. Soc. Lond. 95 (1805) 65-87.
[3] P.-S. Laplace, Théorie de l'action capillaire-Supplément au dixième livre du traité de mécanique céleste, Courcier, Paris, April 23rd, 1806.

[4] A.W. Adamson, A.P. Gast, Physical Chemistry of Surfaces, Wiley, New York, 1997.

[5] A.W. Neumann, J.K. Spelt, Applied Surface Thermodynamics, Dekker, New York, 1996.

[6] A. Bateni, S.S. Susnar, A. Amirfazli, A.W. Neumann, A high-accuracy polynomial fitting approach to determine contact angles, Colloids Surf. A 219 (1-3) (2003) 215-231.

[7] A.F. Stalder, G. Kulik, D. Sage, L. Barbieri, P. Hoffmann, A Snake-based approach to accurate determination of both contact points and contact angles, Colloids Surf. A $286(1-3)(2006)$ 92-103.

[8] M.-W. Yang, S.-Y. Lin, A method for correcting the contact angle from the $\theta / 2$ method, Colloids Surf. A 220 (1-3) (2003) 199-210.

[9] F. Bashforth, J.C. Adams, An Attempt to Test the Theories of Capillary Action, Cambridge University Press, 1883.

[10] Y. Rotenberg, L. Boruvka, A.W. Neumann, Determination of surface tension and contact angle from the shapes of axisymmetric fluid interfaces, J. Colloid Interface Sci. 93 (1983) 169-183.

[11] M. Hoorfar, A.W. Neumann, Axisymmetric drop shape analysis (ADSA) for the determination of surface tension and contact angle, J. Adhes. 80 (8) (2004) 727-743.

[12] P. Cheng, D. Li, L. Boruvka, Y. Rotenberg, A.W. Neumann, Automation of axisymmetric drop shape analysis formeasurement of interfacial tensions and contact angles, Colloids Surf. 43 (1990) 151-167.

[13] C. Atae-Allah, M. Cabrerizo-Vílchez, J.F. Gómez-Lopera, J.A. Holgado-Terriza R. Román-Roldán, P.L. Luque-Escamilla, Measurement of surface tension and contact angle using entropic edge detection, Meas. Sci. Technol. 12 (3) (2001) 288-298.

[14] M.G. Cabezas, A. Bateni, J.M. Montanero, A.W. Neumann, A new drop-shape methodology for surface tension measurement, Appl. Surf. Sci. 238(1-4)(2004) 480-484.

[15] Y.Y. Zuo, C. Do, A.W. Neumann, Automatic measurement of surface tension from noisy images using a component labeling method, Colloids Surf. A 299 (1-3) (2007) 109-116.

[16] R. Ehrlich, An alternative method for computing contact angle from the dimensions of a small sessile drop, J. Colloid Interface Sci. 28 (5) (1968) 5-9.

[17] M.E.R. Shanahan, Profile and contact-angle of small sessile drops-a more general approximate solution, J. Chem. Soc., Faraday Trans. I 80 (1984) 37-45.

[18] H. Park, T. Schoepflin, Y. Kim, Active contour model with gradient directional information: directional snake, IEEE Trans. Circuits Syst. Video Technol. 11 (2) (2001) 252-256.

[19] M. Jacob, T. Blu, M. Unser, Efficient energies and algorithms for parametric snakes, IEEE Trans. Image Process. 13 (9) (2004) 1231-1244.

[20] R. Pruschek, G. Oeljeklaus, V. Brand, G. Haupt, G. Zimmermann, J.S. Ribberink Combined cycle power plant with integrated coal gasification, $\mathrm{CO}$ shift and $\mathrm{CO}_{2}$ washing, Energy Convers. Manage. 36 (6-9) (1995) 797-800.

[21] M. Kanniche, C. Bouallou, $\mathrm{CO}_{2}$ capture study in advanced integrated gasification combined cycle, Appl. Therm. Eng. 27 (2007) 2693-2702.

[22] M. Müller, D. Pavone, M. Rieger, R. Abraham, Hot Fuel Gas Cleaning in IGCC at Gasification Temperature, Fourth International Conference on Clean Coal Technologies, Dresden, Germany, May 2009.

[23] M. Förster, K. Hannes, R. Telöken, Combined cycle power plant with pressurized pulverized coal combustion (PPCC): state of development and perspectives, VGB PowerTech 81 (2001) 30-35.

[24] A.M. Emelyanenko, L.B. Boinovich, The role of discretization in video image processing of sessile and pendant drop profiles, Colloids Surf. A 189(1-3)(2001) 197-202.

[25] M. Unser, Splines: a perfect fit for signal and image processing, IEEE Signal Process Mag. 16 (6) (1999) 22-38.

[26] Wolfram Alpha LLC-A Wolfram Research Company, last checked: December 13th, 2009, URL http://www.wolframalpha.com.

[27] M. Hoorfar, A.W. Neumann, Recent progress in axisymmetric drop shape analysis (ADSA), Adv. Colloid Interface Sci. 121 (2006) 25-49.

[28] J.W. Nowok, J.A. Bieber, S.A. Benson, M.L. Jones, Physicochemical effects influencing the measurements of interfacial surface tension of coal ashes, Fuel 70 (1991) 951-956.

[29] T. Tanaka, M. Matsuda, K. Nakao, Y. Katayama, D. Kaneko, S. Hara, X. Xing, Z. Qiao, Measurement of surface tension of liquid Ga-Base alloys by a sessile drop method, Z. Metallkd. 92 (11) (2001) 1242-1246.

[30] N.M. Dingle, M.T. Harris, A robust algorithm for the simultaneous parameter estimation of interfacial tension and contact angle from sessile drop profiles, J. Colloid Interface Sci. 286 (2005) 670-680.

[31] Homepage of DataPhysics Instruments $\mathrm{GmbH}$, last checked: December 13th 2009, URL http://www.dataphysics.de.

[32] P. Cheng, A.W. Neumann, Computational evaluation of axisymmetric drop shape analysis-profile (ADSA-P), Colloids Surf. 62 (1992) 297-305.

[33] O.I. del Rio, A.W. Neumann, Axisymmetric drop shape analysis: computational methods for the measurement of interfacial properties from the shape and dimensions of pendant and sessile drops, J. Colloid Interface Sci. 196 (1997) $136-147$.

[34] E. Raask, Slag-coal interface phenomena, J. Eng. Power 88 (1) (1966) 40-44.

[35] S.K. Falcone, Ash and Slag Characterization-Final Report for the Period Ending March 31, 1986, Grand Forks, North Dakota, USA, June 1986. 
[36] K.C. Mills, J.M. Rhine, The measurement and estimation of the physical properties of slags formed during coal gasification. 1. Properties relevant to fluid flow, Fuel 68 (1989) 193-200.

[37] J.W. Nowok, J.P. Hurley, A.J. Bieber, The cause of surface tension increase with temperature in multicomponent aluminosilicates derived from coal-ash slags, J. Mater. Sci. 30 (1995) 361-364.

[38] T. Tanaka, M. Nakamoto, R. Oguni, J. Lee, S. Hara, Measurement of the surface tension of liquid $\mathrm{Ga}, \mathrm{Bi}, \mathrm{Sn}$, In and $\mathrm{Pb}$ by the constrained drop method, $\mathrm{Z}$ Metallkd. 95 (9) (2004) 818-822.
[39] E.B. Dismukes, The effect of drop size on the accuracy of surface tension determinations by the sessile drop method, J. Phys. Chem. 63 (1959) 312-314.

[40] G.J. Janz, Molten salts data as reference standards for density, surface tension, viscosity and electrical conductance: $\mathrm{KNO}_{3}$ and $\mathrm{NaCl}$, J. Phys. Chem. Ref. Data 9 (4) (1980) 791-829.

[41] Image-Image Processing and Analysis in Java, last checked: December 13th 2009, URL http://rsbweb.nih.gov/ij. 